\title{
DEVELOPMENT OF A CROSSTALK MEASUREMENT TYPE THREE-DIMENSIONAL DIGITAL COLOR DECODER
}

\author{
Shin-Etsu Ito \\ NHK (Japan Broadcasting Corporation) Broadcasting Center, \\ 2-1, Jinnan 2-chome, Shibuya-ku, Tokyo 150-01 Japan \\ Robert Lowlor and Norio Ebihara \\ Sony Corp. Communication Products Group \\ 4-14-1, Asahi-cho, Atsugi, Kanagawa 243 Japan
}

\begin{abstract}
This paper describes a three-dimensional digital color decoder system to convert composite NTSC or PAL video signals into cross color-free, high picture quality digital component video signals. In this system, the $\mathrm{Y} / \mathrm{C}$ crosstalk is measured by means of three-dimensional crosstalk measurement filters which the authors have newly devised so as to adaptively control the three-dimensional Y/C separation filters.
\end{abstract}

\section{Introduction}

Conventional digital color decoders separate and demodulate composite color video signals into component video signals by using two-dimensional $\mathrm{Y} / \mathrm{C}$ separation circuits or three-dimensional $\mathrm{Y} /$ $\mathrm{C}$ separation circuits. The former system, however, has some problems such that the resolution is degraded and cross-color, crossluminance and dot interference can not be removed sufficiently. While, in the latter system where movements are detected in terms of frame differences, if the input is a still image, Y/C separation is completely done through frame comb type filters, but if it is judged as a moving image, $\mathrm{Y} / \mathrm{C}$ separation is an intra-field two-dimensional operation, and therefore, with an ordinary composite color video signal which involves movements, the same problems as in the former system exist because it retains the aforementioned two-dimensional operation of $\mathrm{Y} / \mathrm{C}$ separation. However, recently, higher picture quality of television signals has been increasingly demanded and EDTV has been put into practical use, and under these circumstances, crosscolor, cross-luminance and dot inter ference, which are caused by incomplete $\mathrm{Y} / \mathrm{C}$ separation and determine the picture quality of composite color video signals, have attracted attention as the existing problems to be solved.
This paper describes a novel crosstalk measurement type threedimensional digital Y/C separation model, which will provide the ultimate solution to these problems, and analyzes the characteristics of the separation filters. Further, it realizes the algorithm as hardware and confirms that the picture quality is improved by a rank of 1.5 at a sight distance of $4 \mathrm{H}$ over the conventional three-dimensional digital color decoders when various composite color video signals including still images, slowly moving images and moving circular zone plates are input actually.

\section{Three-dimensional Power Spectrum of the Composite Color Video Signal (NTSC)}

As shown in Fig. 1, for the composite color video signal of a still image, the $\mathrm{Y}$ signal power spectrum is constructed, on the horizontalvertical spatial frequency axis, as the harmonics synthesis of line spectrums of horizontal and vertical synchronizing frequencies. Moreover, as shwon in Fig. 2, the $\mathrm{C}$ signal power spectrum is distributed, on the vertical-temporal axis, on flat plains of $\pm 15 \mathrm{~Hz}$, and for the entire spatial-temporal axis, it is characteristic that no $\mathrm{Y} /$ $\mathrm{C}$ crosstalk appears.

Thus, for the composite color video signal of a still image, Y/C separation can be done completely by using frame comb type or field comb type filters.

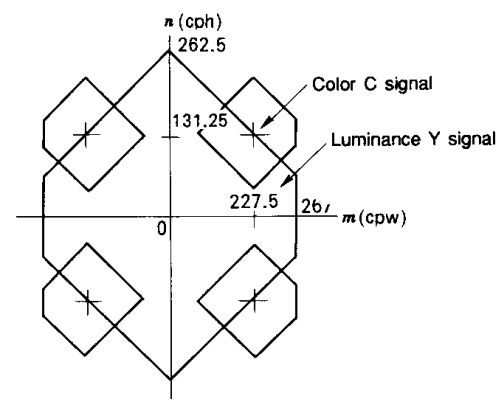

Fig. 1 NTSC, power spectrum on horizontal-vertical axis 


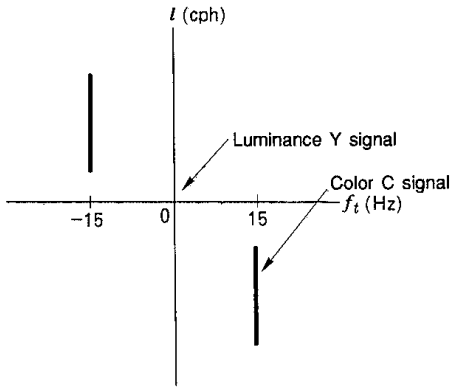

Fig. 2 NTSC, power spectrum on vertical-temporal axis

Fig. 3 and Fig. 4 show typical three-dimensional spectrums of the ordinary composite color video signal which involves movements, as measured by Dubois.

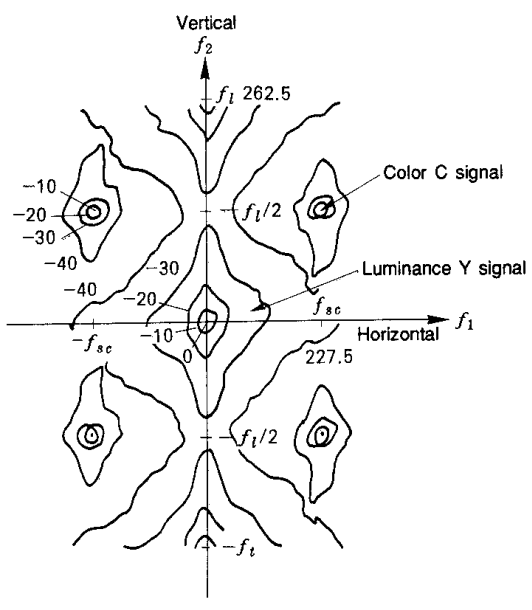

Fig. 3 NTSC, measured power spectrum on horizontal-vertical axis

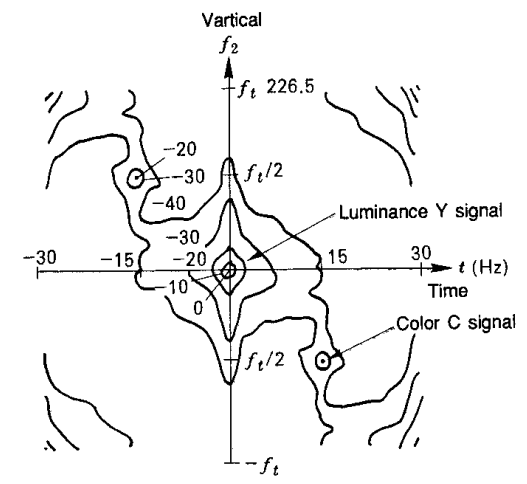

Fig. 4 NTSC, measured power spectrum on vertical-temporal axis
It is seen in Fig. 4 for moving images that unlike in Fig. 2 the power spectrum has a Butterworth spread in the direction of temporal axis so that $Y$ signal and $C$ signal overlap to some extent each other. Therefore, in order to insure sufficient $\mathrm{Y} / \mathrm{C}$ separation, a proper three-dimensional processing is necessary. It is characteristic that when the composite color video signal involves movements, $\mathrm{Y}$ and $\mathrm{C}$ spectrums spread enough to cause crosstalk between $\mathrm{Y}$ and $\mathrm{C}$ signals.

\section{Y/C Separation System}

\subsection{Crosstalk Measuring Filters}

In the three-dimensional digital $\mathrm{Y} / \mathrm{C}$ separation system described in this paper, the crosstalk between $\mathrm{Y}$ and $\mathrm{C}$ signals mentioned above is noted and the crosstalk between $\mathrm{Y}$ and $\mathrm{C}$ signals of each pixel is measured by means of field temporal-spatial oblique comb type and intra-field vertical (line) comb type filters, and the field temporal spatial crosstalk is discriminated as to whether it is $\mathrm{Y}$ based (potential cross color) or $\mathrm{C}$ based (potential cross luminance), and by the resulting control signal, the ratio at which three kinds of $\mathrm{Y} /$ C separation filters (1: inter-field temporal-spatial oblique comb type, 2: inter-field vertical (line) comb type, and 3: horizontal band-pass output) are mixed is adaptively controlled. The greatest feature of the new Y/C separation system is the adoption of this method.

The temporal spatial sampling layout of cross talk measuring filters is shown in Fig. 5.

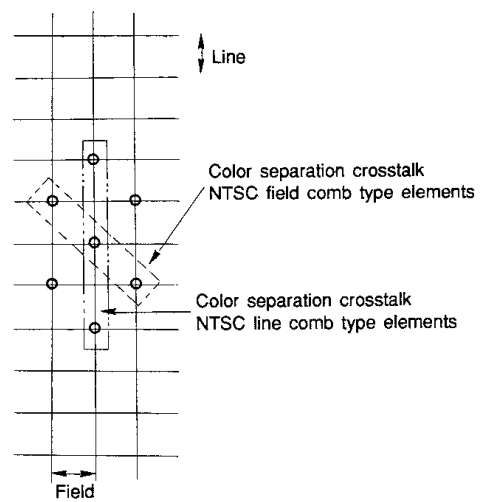

Fig. 5 Layout of sampling points on vertical temporal axis

Fig. 6 shows the pass band characteristics of comb type filters constructed in accordance with the sampling layout mentioned above. By using the field temporal spatial oblique comb type filter and intra-field vertical (line) comb type filter, each $\mathrm{Y} / \mathrm{C}$ crosstalk is measured. The characteristics of the crosstalk measuring filters in this case are given by

$$
\frac{1-e^{j \omega Z T}}{2}
$$

where $\mathrm{T}$ is $263 \mathrm{H}$ for inter-field crosstalk and $1 \mathrm{H}$ for inter-field crosstalk. 


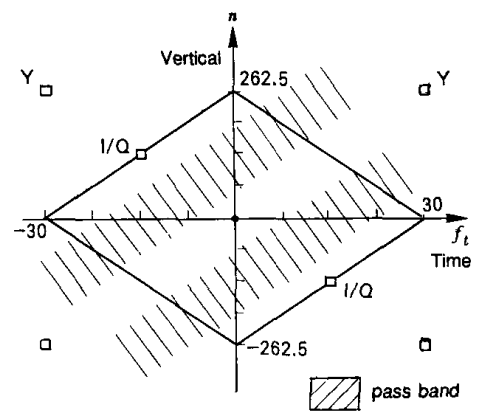

(a) NTSC, inter-field temporal spatial oblique comb type filter

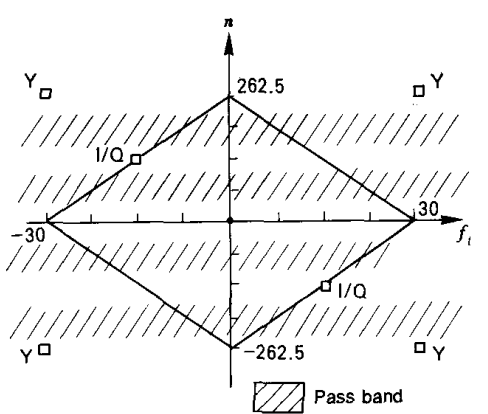

(b) Vertical comb type filter

Fig. 6 Characteristics of crosstalk measuring filters

The filters in Fig. 6 have null points at carrier points of $\mathrm{Y}$ and $\mathrm{C}$, and the crosstalk generating regions construct the pass bands. Thus, the $\mathrm{Y} / \mathrm{C}$ crosstalk caused by movements in the input composite color signal can be measured accurately. In the circuit of Fig. 7, the output of the field temporal spatial oblique crosstalk measuring filter and the output of the intra-field vertical (line) crosstalk measuring filter, for each pixel, are compared with each other.

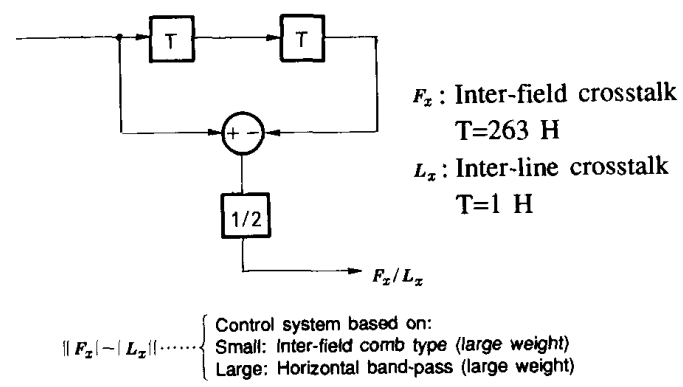

Fig. 7 Block diagram for the measurement of crosstalk measuring filters

If the input composite color signal is judged to have still images or slowly moving images, the weighting on the output of the Y/C separation inter-field comb type filter is increased, or if it has quickly moving images, the weighting on the separation output of the interfield comb type filter is sequentially changed while the output of the inter-field comb type filter is used. Further, if the input image contains a color bar signal or vertical edge component significantly, the Iweighting on the horizontal band-pass filter is increased. The related coefficients have been kept in ROM memory for real-time processing so that the image after $\mathrm{Y} / \mathrm{C}$ separation is not degraded in its characteristics by varying movements.

Fig. 8 shows a block diagram for controlling three kinds of $\mathrm{Y} /$ C separation filters according to the magnitude of crosstalk.

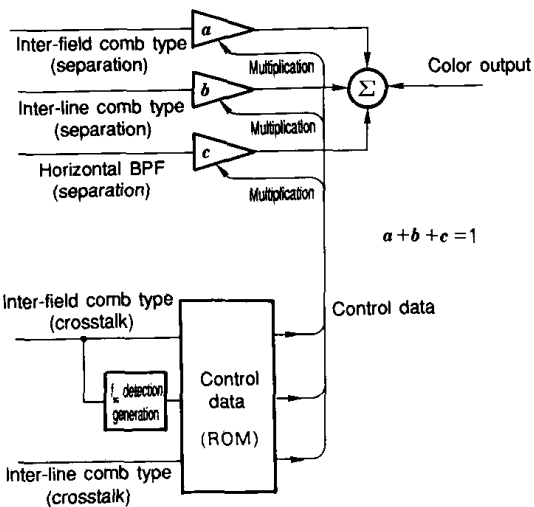

Fig. 8 Block diagram of filter control circuit

However, for an all spatial frequency pattern which involves movements such as a fountain zone plate image, the afore-mentioned two kinds of crosstalk measuring filters alone are insufficient and result in degraded picture quality as shown in Photo 1.

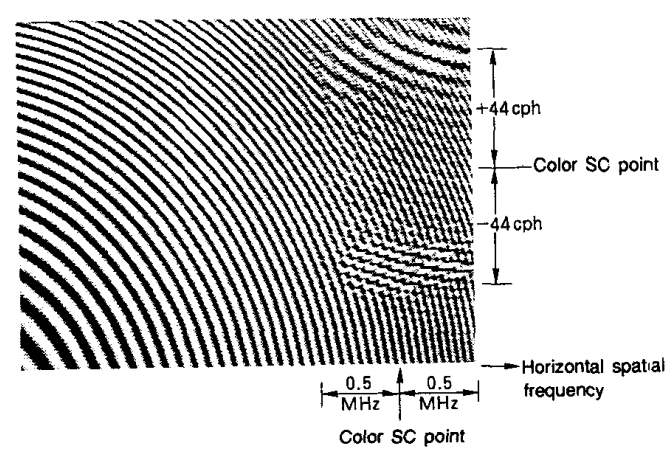

Photo 1 NTSC, regenerated $Y$ signal of a fountain zone plate image 
The degradation in this case is caused because the crosstalk measuring filters are not completely three-dimensionalized, that is, the inter-field temporal-spatial oblique crosstalk filters are not provided with a function to discriminate the measured crosstalk as to whether it is $\mathrm{Y}$ based (potential cross color) or $\mathrm{C}$ based (potential cross luminance). On the other hand, the horizontal frequency component of the $\mathrm{C}$ based crosstalk has been known to be near the subcarrier frequency, and threrefore, it is possible to do crosstalk evaluation by placing a horizontal frequency detector in the interference path. This crosstalk evaluation method is very effective to determine which $\mathrm{Y} / \mathrm{C}$ separation filter is most suited for the contents of the instantaneous spectrum of the composite signal.

\subsection{Horizontal Frequency Detection Algorithm}

The algorithm of the afore-mentioned new third crosstalk evaluating filter is described below. For the derivation of this algorithm, refer to Appendix.

Now, let $S(t)$ be a temporal domain sine wave of frequency $\mathrm{f} \mathrm{Hz}$, assume that in order to prevent aliasing of $S(t)$, sampling is done at $f_{c k} \mathrm{~Hz}$ in the range of $f_{c k} \gg 2 f$, and let $S(n T)$ denote the sampled signal as the result, where $n$ is an integer and $T=1 / f_{c k}$ second.

Let the three consecutive samples of $\mathrm{S}(\mathrm{nT})$ be as follows:

$$
\begin{aligned}
& S_{1}=A \sin \left(\omega t+\phi_{1}\right) \\
& S_{2}=A \sin \left(\omega t+\phi_{1}+\phi_{s}\right) \\
& S_{3}=A \sin \left(\omega t+\phi_{1}+2 \phi_{s}\right)
\end{aligned}
$$

where $A$ is the amplitude of the sine wave, $\omega=2 \pi f, \emptyset_{1}$ is an arbitrary phase shift with respect to the first sample, and $\emptyset_{\mathrm{s}}$ is the phase change of the sine wave for one clock period and

$$
\phi_{s}=2 \pi \frac{f}{f_{c k}}
$$

In order to derive the frequency detection algorithm, assume that A, $\omega$ and $\varnothing$ are unknown. By algebraic rearrangement of Eqs. (1) through (3), we can derive the necessary and sufficient condition for Eq. (4). This condition is given by

$$
S_{1}-2 S_{2} \cos \phi_{s}+S_{3}=0
$$

For the derivation of this equation, refer to Appendix.

A practical configuration in accordance with Eq. (5) is the trap FIR filter shown in Fig. 9.

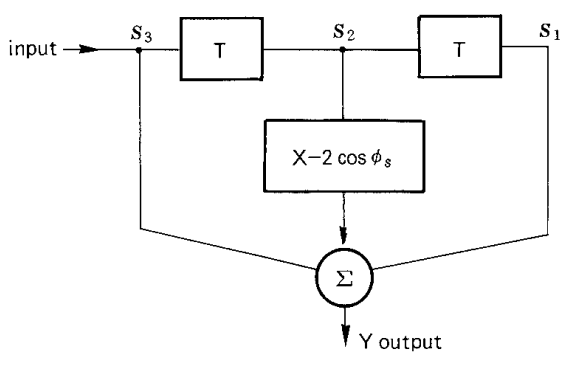

Fig. 9 Filter configuration

This filter has the following amplitude characteristics.

$$
\begin{aligned}
Y & =e^{-j \omega T}-2 \cos \phi_{s}+e^{+j \omega T} \\
& =2\left\{\cos \omega T-\cos \phi_{s}\right\}
\end{aligned}
$$

Fig. 10 shows the corresponding filter conversion characteristics.

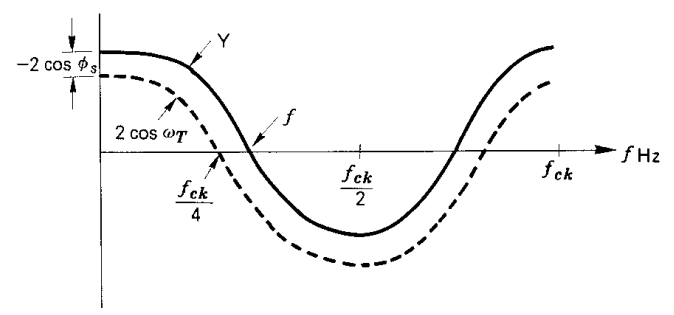

Fig. 10 Filter characteristics

As shown in Fig. 10, the amplitude characteristic crosses the axis of $\mathrm{Y}=0$ at frequency $\mathrm{f} \mathrm{Hz}$.

The fact that when a non-zero signal is applied to the filter input, no output is provided indicates that the frequency of the input signal is $\mathrm{f} \mathrm{Hz}$.

In this algorithm, the frequency detector operates best as such in the domain of $\mathrm{f}_{\mathrm{ck}} / 4$ where $2 \cos \omega \mathrm{T}$ is steepest (actually, it works effectively in the range of $0<\mathrm{f}<\mathrm{f}_{\mathrm{ck}} / 2$ ).

This frequency detection algorithm is used for the third $f_{s c}$ detection filter of Fig. 7 in this crosstalk measurement type threedimensional digital color decoder. It is just the novel and most important part in the whole $\mathrm{Y} / \mathrm{C}$ separation circuits. It makes it possible to discriminate the output of the field temporal-spatial oblique crosstalk measuring filter as to the whether it is $\mathrm{Y}$ based (potential cross color) or $\mathrm{C}$ based (potential cross luminance), and hence to measure the complete three-dimensional crosstalk on the temporalspatial and thereby remedy the degraded picture quality in Photo 1 . 


\subsection{Y/C Separation Filter}

The Y/C separation three-dimensional filter shares the temporalspatial sampling point in Fig. 5 with the afore-mentioned cross color measuring filter.

Fig. 11 and Fig. 12 show the configuration of the inter-field temporal-spatial oblique Y/C separation filter and the intra-field vertical (line) comb type filter, respectively.

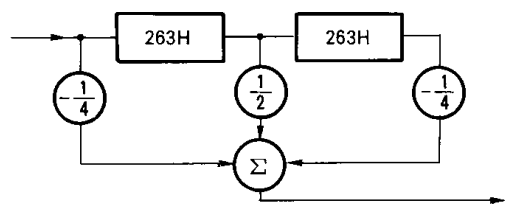

Fig. 11 NTSC, block diagram of the temporal-spatial oblique comb filter

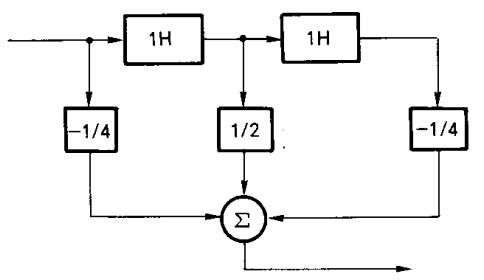

Fig. 12 NTSC, block diagram of the intra-field vertical comb filter

The two kinds of filters having the above characteristics are combined with the horizontal band-pass filter, and the output signals from the resulting three kinds of $\mathrm{Y} / \mathrm{C}$ separation filters are adaptively controlled by using the control data generated from the measured crosstalk values described in the preceding paragraph, and then they are synthesized to result in color signal (C). On the other hand, luminance signal ( $\mathrm{Y}$ ) can be obtained by deriving this $\mathrm{C}$ signal from
Fig. 13 and Fig. 14 show the characteristics of the respective filters.

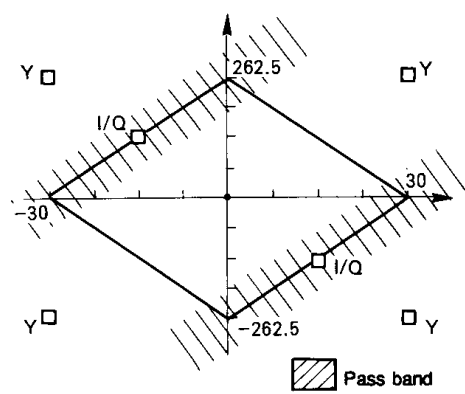

Fig. 13 NTSC, characteristics of the temporal-spatia oblique comb filter

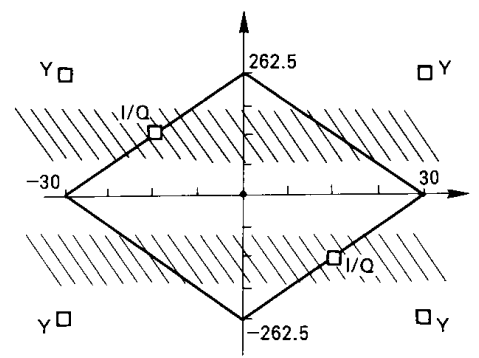

Fig. 14 NTSC, characteristics of the intra-field vertical comb filter

the input composite color signal which is in the same timing.

\subsection{Block Diagram of the Color Decoder}

Fig. 15 shows the block diagram of this three-dimensional digital color decoder with special emphasis placed on $\mathrm{Y} / \mathrm{C}$ separation circuits.

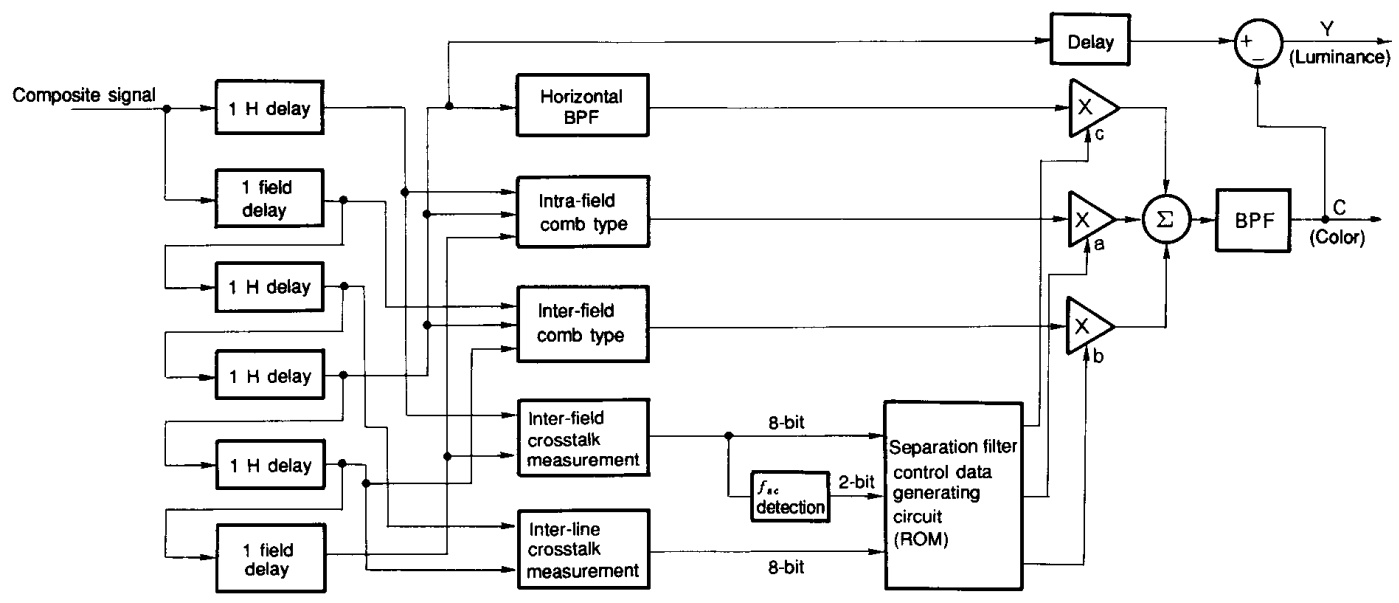

Fig. 15 Block diagram of the three-dimensional digital color decoder 


\section{Extension to PAL Video Signals}

4.1 Temporal-spatial Power Spectrum of PAL Video Signals

Descriptions so far given refer mainly to the $\mathrm{Y} / \mathrm{C}$ separation processing of NTSC composite color video signals. They can be extended to PAL Video signals as follows.

It is well known that in the PAL color television system the carrier of color difference signal $\mathrm{u}$ is $180^{\circ}$ altered in phase by line in order to reduce color transmission distortion DP. As a result, the carrier of color difference signal $u$ and the carrier of color difference signal $v$ are offset by $1 / 2$ line with respect to each other and by 1 / 4 line with respect to $Y$ signal. The PAL video signal having the relationship of frequency interleaving described above has the temporal-spatial power spectrum as shown in Fig. 16.

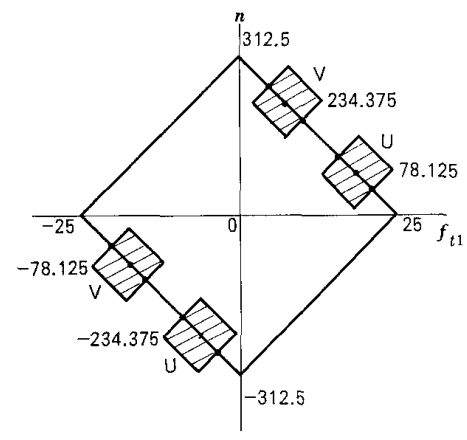

Fig. $16 \mathrm{PAL}$, power spectrum on temporal-spatial axis

In the color decoder of this paper, this power spectrum of the $\mathrm{PAL}$ system is taken into consideration, and cross color measuring filters and $\mathrm{Y} / \mathrm{C}$ separation filters are constructed so that the $\mathrm{Y} / \mathrm{C}$ separation hardware may be compatible with the NTSC system.

Since the PAL video signal has the temporal-spatial power spectrum as shown in Fig. 16, crosstalk measuring filters having the characteristics of Fig. 17 are used.

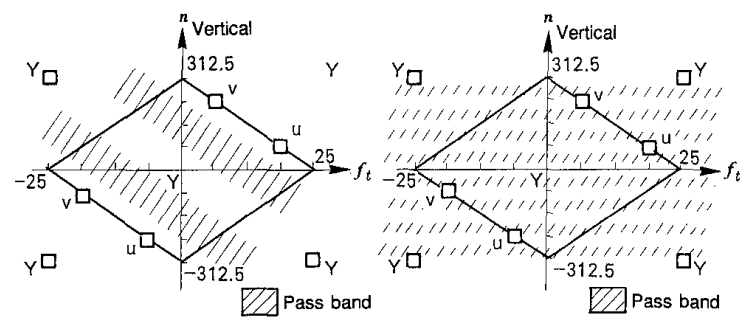

(a) Inter-field temporal-spatial (b) Vertical comb type filter oblique comb type filter

Fig. 17 PAL, characteristics of crosstalk measuring filters

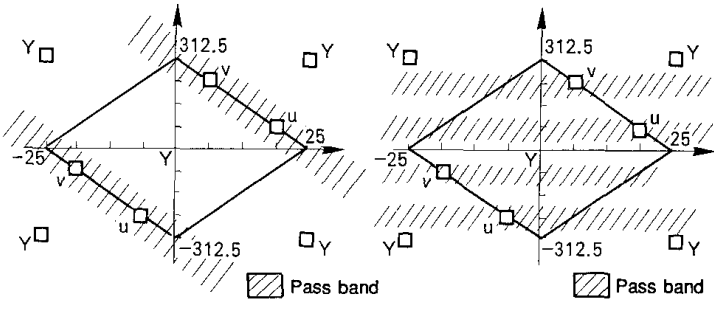

(a) Inter-field temporal-spatial (b) Vertical comb type filter oblique comb type filter

Fig. 18 PAL, characteristics of $\mathrm{Y} / \mathrm{C}$ separation filters

These filters can be constructed to have $\mathrm{T}=312 \mathrm{H}$ for inter-field crosstalk and $\mathrm{T}=2 \mathrm{H}$ for intra-field (inter-line) crosstalk in Fig. 7 which refers to NTSC. On the other hand, Y/C separation filters have characteristics as shown in Fig. 18. These filters can be constructed to be $312 \mathrm{H}$ and $2 \mathrm{H}$, respectively in Fig. 11 and Fig. 12, which refer to NTSC. Since the filter configuration is the same for NTSC and PAL as described above, the new digital color decoder system can be used in either system merely by touch operation.

\section{Color Demodulation Circuit}

The color signal after $\mathrm{Y} / \mathrm{C}$ separation is subsequently demodulated through a color demodulation circuit of Fig. 19 into component $\mathrm{Y}, \mathrm{R}-\mathrm{Y}$ and $\mathrm{B}-\mathrm{Y}$ signals.

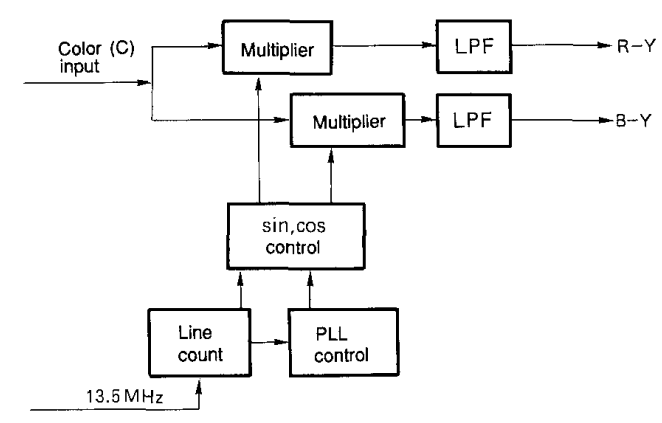

Fig. 19 Block diagram of color demodulation circuit

Throughout the equipment, the system clock frequency is 13.5 $\mathrm{MHz}$ which is completely conforming to CCIR Rec-601, and is generated by line locking with the input video signal. 


\section{Design Specifications for the Crosstalk Measurement Type Three-Dimensional Digital Color Decoder}

Table 1 shows the specifications for this digital color decoder in design stage.

Table 1 Specifications of the digital color decoder

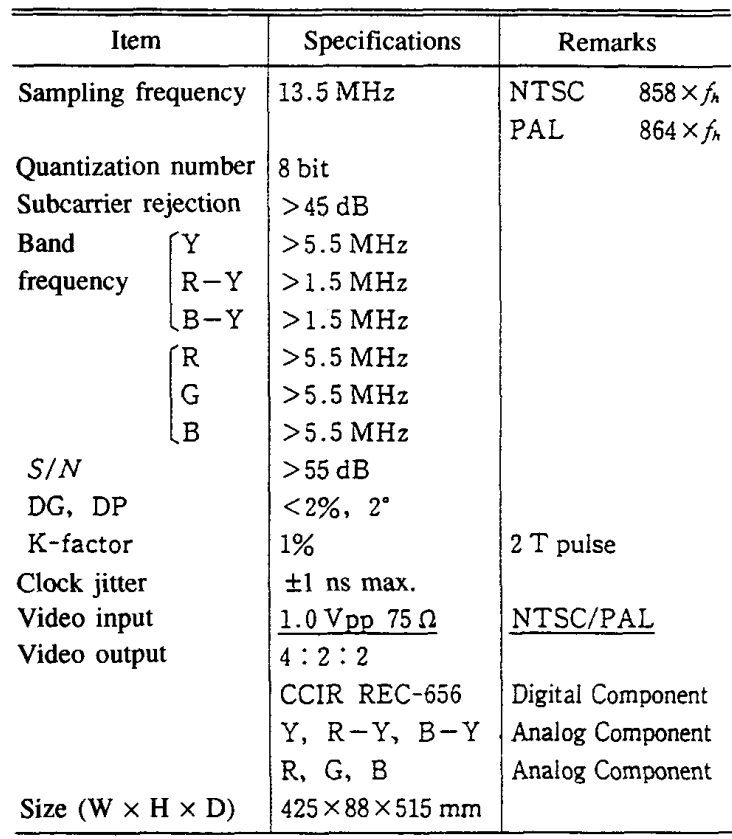

After development, it was confirmed by various tests such as zone plate, sweep, color bar, etc. that the development target shown in this table had been achieved successfully.

\section{Picture Quality of the Crosstalk Measurement Type Three-Dimensional Digital Color Decoder}

\subsection{Still Zone Plate Video Signals}

When the NTSC still zone plate video signal is input to this digital color decoder, luminance signal $(\mathrm{Y})$ as shown in Photo 2 is output after $\mathrm{Y} / \mathrm{C}$ separation.

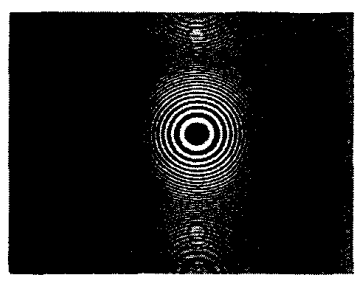

Photo 2 Regenerated $Y$ signal of a still zone plate image

As seen in this photo, cross color-free, dot interference-free video signals are obtained from inter-field comb type filters.

\subsection{Moving Zone Plate Video Signals}

When the NTSC video signal which involves a fountain zone plate image is input to the digital color decoder, $\mathrm{Y}$ signal as shown in Photo 2 is output after $\mathrm{Y} / \mathrm{C}$ separation.

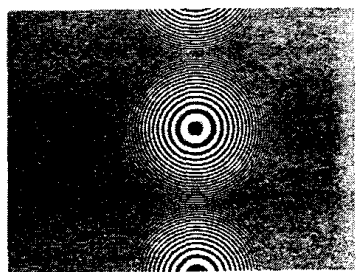

Photo 3 NTSC, regenerated $Y$ signal of a fountain zone plate image

This photo is free from any flaws caused by maldiscrimination in Photo 1. Even with the input signal of a moving image, $\mathrm{Y} / \mathrm{C}$ separation is done accurately and ideal characteristics almost free from cross color and other interferences are obtained. Further, the use of inter-field comb type filters prevents generation of any interference such as frame lag. Moreover, in the evaluation with horizontally moving zone plates, moving images of black-white oblique striped patterns, etc., any interference such as cross color was not noticed. With ordinary video signals, the picture quality was improved by a rank of 1.5 at a sight distance of $4 \mathrm{H}$ over the conventional three-dimensional digital color decoders. Photo 4 shows regenerated $\mathrm{Y}$ signal after separation of a horizontally moving zone plate image.

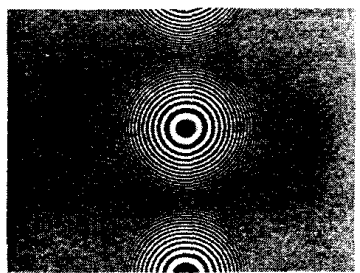

Photo 4 NTSC, regenerated Y signal of a horizontally moving zone plate image

\section{Conclusion}

For the composite video signal, an ingenious way of frequency interleaving multiplex is applied for effective utilization of frequency. However, it is very difficult to separate $\mathrm{Y} / \mathrm{C}$ spectrums completely, and in the receivers for demodulated NTSC video signals or PAL video signals, cross color, cross luminance and dot interference occur and cause the picture quality to be degraded. These problems existed to be solved.

The new digital color decoder system presented in this paper offers a reasonable solution to these problems. The authors believe that it has established a form of high picture quality reception and high picture quality decoding. If it is realized in smaller size and lighter weight and is applied to home receivers in the future, it will be possible to enjoy significantly improved picture quality. 


\section{ACKNOWLEDGMENT}

The authors express their deep thanks to Mr. Yoshinobu Ohba, senior master researcher of NHK Science \& Technology Laboratories for his consistent instructions, and $\mathrm{Mr}$. Takeshi Ogawa, general director and Mr. Masato Kawaguchi, deputy director, both of NHK Engineering Development Center, for their advice in detail. Further, the authors thank R \& D group Sony Broadcast and Communications in England and Business \& Professional Group in Japan for their assistance in hardware configuration.

\section{APPENDIX}

$$
\begin{aligned}
S_{1} & =A \sin \left(\omega t+\phi_{1}\right) \\
S_{2} & =A \sin \left(\omega t+\phi_{1}+\phi_{s}\right) \\
S_{3} & =A \sin \left(\omega t+\phi_{1}+2 \phi_{s}\right) \\
\therefore \quad & S_{3}=A \sin \left\{\left(\omega t+\phi_{1}+\phi_{s}\right)+\phi_{s}\right\} \\
& =A \sin \left(\omega t+\phi_{1}+\phi_{s}\right) \cos \phi_{s}+A \cos \left(\omega t+\phi_{1}+\phi_{s}\right) \sin \phi \\
& =S_{2} \cos \phi_{s}+\sqrt{A^{2}-S} \\
S_{2} & =S_{1} \cos \phi_{s}+\sqrt{A^{2}-S_{1}^{2}} \sin _{s} \\
\therefore \quad & \\
A^{2} & =\frac{1}{\sin ^{2} \phi_{s}}\left(S_{2}-S_{1} \cos \phi_{s}\right\}^{2}+S_{1}^{2} \\
\therefore \quad & \\
S_{3} & =S_{2} \cos \phi_{s}+\left\{S_{1}^{2}-S_{2}^{2}+\frac{1}{\sin ^{2} \phi_{s}}\left(S_{2}-S_{1} \cos \phi_{s}^{2}\right)\right\}^{1 / 2} \sin \phi_{s} \\
& =S_{2} \cos \phi_{s}+\sqrt{\left(S_{1}-S_{2} \cos \phi_{s}\right)^{2}} \\
& =S_{2} \cos \phi_{s} \pm\left(S_{1}-S_{2} \cos \phi_{s}\right)
\end{aligned}
$$

Similarly,

Here, if the positive sign is taken, then $S_{3}=S_{1}$. This is the natural result of the following relation and has no meaning.

$$
\cos \phi_{s} \equiv \cos \left(-\phi_{s}\right)
$$

Moreover, it corresponds to the fact that the phase change from $S_{2}$ to $S_{3}$ is equal in magnitude but reverse in direction to the phase change from $S_{1}$ to $S_{2}$. That is,

$$
S_{3}=A \sin \left(\omega t+\phi_{1}+\phi_{s}-\phi_{s}\right)=S_{\mathbf{I}}
$$

Here, if the negative sign is taken, then

$$
S_{1}-2 S_{2} \cos \phi_{s}+S_{3}=0
$$

This is the result being sought.

\section{REFERENCES}

[1] D. Creed, S. Ito and N. Ebihara, "An Advanced NTSC Digital Decoder," 130th SMPTE Technical Conference, October, 1988

[2] S. Ito, et al., "Development of Improved Three Dimensional Digital Color Decoder Conforming to CCIR REC-601," ITEJ J. of Japan, No. 9, 1990

[3] Dubios, et al., "3-Dimensional Spectrum and Processing of Digital NTSC Color Signals," SMPTE J. April, 1982
[4] Y. Nakajima, et al., "Improvement of Picture Quality for NTSC and PAL Systems by Digital Signal Processing," IEEE Trans. Consumer, Vol. CE31, No. 4, November, 1985

[5] G. Gerhard et al., "Improved PAL Using a Combination of NTSC, SECAM, and PAL," SMPTE J., 1986

[6] C. H. Strolle, "Cooperative Processing for Improved NTSC Chrominanc/Luminance Separation," SMPTE J., August, 1986

[7] Mikhail Tsinberg et al.. "A Two Dimensional Adaptive Digital Decoder for Color Television Signals," IEEE Trans, Consumer, Vol. CE-33, No. 3, August, 1987

[8] Y. Farodja et al., "Improving NTSC to Achieve Near-RGB Performance," SMPTE J., August, 1987

[9] W. Schreiber, "Improved Television System: NTSC and Beyond," SMPTE J., August, 1987

[10] Y. C. Faroudja, "NTSC and Beyond," IEEE Trans. Consumer, Vol. 34, No. 1, February, 1988

[11] Detlef Teichner, "Three-Dimensional Pre and Post Filtering for PAL Signals," IEEE Trans. Consumer, Vol. 34, No. 1, February, 1988

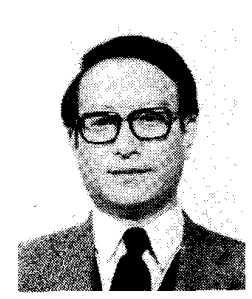

Shin-etsu Ito was born in Akita Prefecture, Japan, on March 21, 1950. He received the B.S.E.E. degree from the University of Akita, in 1974. In the same year, he joined NHK (Japan Broadcasting Corporation), Japan, and through the Engineering Department of the Production Engineering Bureau, he moved in 1982 to the Program Facilities Department of the Engineering Headquarters where he was engaged in the design and development of digital data transmission apparatus and computer communication systems. In 1984, he turned to the Development Engineering Department of the same Headquarters, and since then, has been engaged in the design and development mainly of digital video signal processing systems, digital magnetic recording systems, satellite digital transmission systems, etc.

Mr. Ito is a member of IEEE of U.S.A., SMPTE of U.S.A., IEICE of Japan, and ITEJ of Japan.

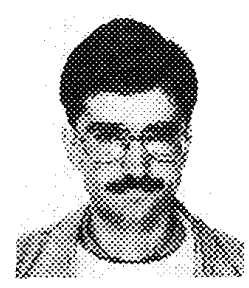

Robert Lowlor was graduated from Kelvin Street College (Ireland), Electrical Engineering Group in 1984. In the same year, he joined Sony Broadcast (England) and in R \& D Group, engaged in the development of digital video signal processing. Now, in Sony Corporation (Japan), he is engaged in the development and design of video apparatus for business use.

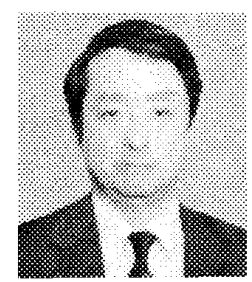

Norio Ebihara was graduated from Keio University, Master Course in 1965. He joined Oki Electric Industry Co., Ltd., then moved to Sony Corporation, and through the Central Research Laboratories and the Video Division, now in the System Division, he is engaged in the development and design of digital image processing equipment. 